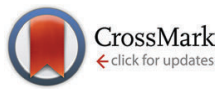

Cite this: DOI: 10.1039/c6sm01647b

\title{
Guest-matrix interactions affect the solvation of cyclodextrin-based polymeric hydrogels: a UV Raman scattering study
}

\author{
B. Rossi, ${ }^{\star a b}$ V. Venuti, ${ }^{c}$ F. D'Amico, ${ }^{a}$ A. Gessini, ${ }^{a}$ A. Mele, ${ }^{d}$ C. Punta, ${ }^{d}$ L. Melone, ${ }^{d e}$ \\ V. Crupi, ${ }^{C}$ D. Majolino ${ }^{C}$ and C. Masciovecchio ${ }^{a}$
}

\begin{abstract}
The focus of the present work is to shed light on possible modifications of the molecular properties of polysaccharide hydrogels induced by the establishment of specific non-covalent interactions during the loading of a guest compound inside the gel phase. With this aim, a case study of the encapsulation of caffeine (Caf) inside cyclodextrin-based hydrogels, namely, cyclodextrin nanosponges (NS), is systematically investigated here by using UV Raman scattering experiments. The UV Raman spectra of the hydrogels, analysed as a function of temperature, concentration of the guest molecule loaded in the gel phase and $\mathrm{pH}$, prove particularly informative both on the structural rearrangements of the hydrophobic/hydrophilic groups of the polymeric network and on the breaking/formation of specific guest-matrix interactions. Analysis of the temperature dependence of dynamical parameters, i.e., the dephasing time associated with specific vibrational modes of the polymer backbone, enables the proposal of a molecular picture in which the loading of Caf in NS hydrogels tends to favour access of the water solvent to the more hydrophobic portions of the polymer matrix, which is in turn reflected in a marked increase in the solvation of the whole system. The achievements of this work appear of interest with respect to the design of new possible strategies for controlling the diffusion/release of bioactive molecules inside hydrogel networks, besides corroborating the potential of UV Raman scattering experiments to give new molecular insights into complex phenomena affecting hydrogel phases.
\end{abstract}

Accepted 30th September 2016

DOI: $10.1039 / \mathrm{c} 6 \mathrm{sm} 01647 \mathrm{~b}$

www.rsc.org/softmatter attractive for a wide range of technological applications. For example, the physiochemical similarity of hydrogels to the native extracellular matrix suggested the use of these materials as scaffolds for tissue engineering and regenerative medicine. ${ }^{5-7}$ Indeed, synthetic matrices where cells can grow and interact with the environment in all three dimensions allow the in vivo behaviour of cells to be mimicked more efficiently than in previous methods based on the $2 \mathrm{D}$ growth of cells. Other examples include wound dressings, ${ }^{8}$ super-adsorbent systems ${ }^{9}$ and many suitable formulations for drug delivery. ${ }^{10-12}$ Besides these practical aspects, hydrogels are interesting model systems for studying the role of hydrophobic and hydrophilic interactions in determining the gelling behaviour of biomacromolecules. This has been recently shown in the case of polysaccharidebased hydrogels, which are characterized by the presence in their polymeric backbone of both hydrogen bond donor and acceptor groups, ${ }^{13-15}$ around which water can be rearranged in different ways.

It is well known that important chemical and physical properties of hydrogels are determined, at the molecular level, by both structural features of the hydrogel matrix (e.g., the density of cross-links) and intermolecular interactions such as 
hydrophobic associations and hydrogen bonds. In principle, the water retention capability and mechanical stability of a hydrogel can be controlled by tuning the porous structure of the gel matrix and the chemical affinity of the polymeric network towards the aqueous environment. ${ }^{2}$ Special attention has been paid to the effect of molecular architecture and intermolecular interactions on the response of hydrogels to external stimuli such as variations in temperature or $\mathrm{pH}$. This is also due to the growing use of hydrogels in fields of high social impact that require the continuous design and development of 'smart' hydrogels that are capable of interacting with their environment in a pre-programmed and intelligent manner. ${ }^{16-19}$ The mechanisms governing the loading and release of drugs or active agents from a hydrogel matrix are a current field of investigation, as well as the assessment of the advantages and limitations of hydrogels as carrier systems. ${ }^{20}$ Several factors contribute to the overall performance of hydrogels as absorption/release systems such as the viscosity of the gel phase, the transport properties of the small molecules or macromolecules trapped in the gel and the porosity of the matrix. On the other hand, it has been shown how non-covalent interactions between the polymeric matrix and a guest compound confined inside the gel may alter the properties of hydrogels, thus compromising their final release behaviour. ${ }^{21}$

In the past few years, our group has developed and characterized a class of polymeric materials derived from natural cyclodextrins (CD), which are referred to as cyclodextrin nanosponges (NS). The synthesis of NS is based on the covalent cross-linking of CD with suitable cross-linking agents. ${ }^{22}$ Many NS display interesting swelling behaviour in water or aqueous solutions, leading to the formation of hydrogels. These hydrogels have many advantageous features with respect to other hydrogel formulations, including low toxicity and good biocompatibility. The capability of NS to provide innovative solutions for the delivery and controlled release of drugs has been recently reviewed by different authors. ${ }^{23-26}$ Additional uses of NS in agriculture ${ }^{27}$ and environmental control ${ }^{28}$ are also reported in the literature.

A description of the gelling behaviour of NS hydrogels in terms of their structural and dynamical properties has been provided in some recent works, ${ }^{13-15,29}$ mainly via the analysis of vibrational spectra. These studies suggest that the hydration of NS and the viscosity of the gel phase can be tuned by controlling specific factors during the synthesis of $\mathrm{NS}^{15,30}$ and by regulating the hydration level ${ }^{14}$ and the $\mathrm{pH}$ of the system. ${ }^{13,29}$ The latter parameter has also been found to affect the thermoactivation of NS hydrogels, ${ }^{13,29}$ which at high temperatures display stronger solvation of the more hydrophobic parts of the polymer backbone. ${ }^{29}$ This effect has been efficiently monitored by following the temperature dependence of the dephasing time associated with the vibrational modes involving specific moieties present in the polymer matrix of NS. ${ }^{29}$

In this general context, our main interest lies in studying the mechanisms of the loading, diffusion and release of guest compounds confined inside NS hydrogels. A pilot study on ibuprofen-loaded NS hydrogels pointed out how the transport<smiles>Cn1c(=O)c2c(ncn2C)n(C)c1=O</smiles>

Scheme 1 Structure of caffeine.

properties of ibuprofen in the gel phase are strongly dependent on the structural properties of the network of NS. ${ }^{31}$

The present work is mainly aimed at studying how the molecular properties of NS hydrogels can change in response to non-covalent interactions with caffeine (Caf), which is used here as a model guest compound.

The case study of Caf confined in NS hydrogels was undertaken here by using UV Raman scattering experiments, with the aim of gaining insights into the fundamental molecular interactions that occur in the processes of loading and diffusion in the NS gel phase. Caffeine (1,3,7-trimethylxanthine, Scheme 1) was selected as a molecular probe of guest-matrix interactions owing to its small and relatively simple structure and in view of its potential applications in pharmaceutical ${ }^{32,33}$ and cosmetic formulations. $^{34,35}$ Because Caf exhibits a stimulating effect on the cutaneous microcirculation but poor skin penetration, there is wide interest in developing new promising carriers for the controlled topical administration of caffeine in cosmetic formulations. ${ }^{36}$

UV Raman scattering investigations of NS hydrogels loaded with Caf were carried out as a function of temperature and guest concentration with the aim of studying the effect of guest-matrix interactions on the solvation behaviour of NS polymers. ${ }^{13,29}$ This process is particularly informative on the structural rearrangements of the hydrophobic/hydrophilic groups of NS and on the breaking/formation of specific non-covalent interactions between chemical groups of Caf and the polymer skeleton of NS.

The study was performed on two types of NS, which were obtained by varying during the synthesis the molar ratio of cyclodextrin to the cross-linker. This choice was made on the basis of structural knowledge of the polymeric network of $\mathrm{NS}^{14,15,30}$ and by considering previous results for the diffusivity of guest molecules inside NS gels. ${ }^{31}$

Finally, the analysis of the thermal dependence of dynamical Raman parameters such as the dephasing time can be correlated with the mechanism of the release of Caf from NS hydrogels in response to external stimuli such as variations in temperature.

\section{B Experimental}

\section{A Synthesis of nanosponge polymers}

Nanosponge polymers were prepared according to the standard procedure as previously reported. ${ }^{13,29}$ In brief, $\beta$-cyclodextrin $(\beta-\mathrm{CD})$ was dissolved in anhydrous DMSO and in the presence of anhydrous $\mathrm{Et}_{3} \mathrm{~N}$ was reacted with the cross-linking agent pyromellitic anhydride (PMA) at room temperature for 3 hours under intensive stirring. PMA was added at molecular ratios of 
$1: 4$ and $1: 8$ with respect to the monomer $\beta$-CD. In the following, the corresponding polymers will be referred to as $\beta$-CDPMA14 and $\beta$-CDPMA18, where the digits refer to the molar ratio of $\beta$-CD to PMA used in the synthesis. After the reaction procedure, the obtained polymers were crushed in a mortar, purified by washing with $0.2 \mathrm{M} \mathrm{HCl}(\mathrm{aq})$ (3 times) and deionized water (5 times), and finally dried under vacuum to afford a homogeneous powder.

Previous works ${ }^{14,15}$ on $\beta$-CDPMA14 and $\beta$-CDPMA18 nanosponges showed that these polymers could form, after swelling with water or aqueous solutions, transparent, homogeneous gels in which guest molecules can efficiently diffuse. ${ }^{31}$

\section{B Loading of caffeine into NS hydrogels}

Caffeine (Caf, $\mathrm{C}_{8} \mathrm{H}_{10} \mathrm{~N}_{4} \mathrm{O}_{2}$ ) was purchased from Aldrich and used without further purification. Three sequential steps were followed for the loading of Caf into NS hydrogels. (1) Suitable amounts of dry Caf and dry NS polymer were weighed and accurately mixed. (2) A hydrating solution was prepared by adding to double-distilled water a suitable amount of $\mathrm{Na}_{2} \mathrm{CO}_{3}$ $(15 \% \mathrm{w} / \mathrm{w})$ in order to regulate the final value of the $\mathrm{pH}$ in the gel phase, following the procedure for the preparation of NS hydrogels already used in previous works. ${ }^{13,29,31}$ (3) Finally, a Caf-loaded NS hydrogel was obtained by adding to the dry mixture from step (1) a measured quantity of the solution from step (2) in order to achieve the desired level of hydration of $h=4$ (where $h$ is defined as the weight ratio of water to NS). After this procedure, a perfectly homogeneous and transparent gel without any visible phase separation or solid particles was obtained in about one hour. The final concentration of Caf loaded in the gel phase $\left(C_{\mathrm{Caf}}, \mathrm{mg} \mathrm{mL}^{-1}\right)$ was defined as the ratio between the weight of Caf used in step (1) and the volume of the hydrating solution used in step (3). The relative content of the guest compound with respect to the host nanosponge was expressed as the weight/weight ratio between Caf and NS used in step (1). The final measured value of the $\mathrm{pH}$ in the gel loaded with Caf was about 9.2. The decision to study Caf-loaded gels of nanosponges in basic conditions was dictated by previous experimental results ${ }^{13,29,31}$ that suggest an increase in the swelling capability of the NS polymer matrix with respect to neutral or acid conditions.

\section{UV Raman scattering measurements and fitting procedure}

UV Raman scattering measurements were carried out at the BL10.2-IUVS beamline at the Elettra synchrotron laboratory in Trieste with the experimental setup described by D'Amico et al. ${ }^{37}$ Hydrogel samples were freshly prepared and placed into optical quartz cuvettes for the measurements. The sample temperature was controlled during the acquisition of spectra and set at different values in the range of 297 to $364 \mathrm{~K}$. Polarized parallel $\left(I_{\mathrm{VV}}\right)$ and depolarized $\left(I_{\mathrm{HV}}\right)$ UV Raman spectra were excited at $266 \mathrm{~nm}$ and recorded in a back-scattered geometry using a triplestage spectrometer (TriVista, Princeton Instruments). The experimental resolution was set at $5 \mathrm{~cm}^{-1}$ in order to ensure sufficient resolving power and count rate. In order to prevent possible photodecomposition of the gels resulting from exposure to
UV radiation, the sample cell was subjected to slow continuous spinning during the recording of measurements in order to vary the volume of the sample that was illuminated by the exciting radiation beam. The isotropic Raman intensity was calculated according to the relation:

$$
I_{\mathrm{ISO}}=I_{\mathrm{VV}}-\frac{4}{3} I_{\mathrm{HV}}
$$

The isotropic Raman spectra were fitted using a combination of Kubo-Anderson functions, following the steps described in detail by Rossi et al. ${ }^{14,29}$ For Raman peaks that were found to be approximated by a Lorentzian function, the corresponding dephasing time $\tau_{\mathrm{deph}}$ was computed using the relation:

$$
\tau_{\mathrm{deph}}=\frac{1}{\pi \Gamma}
$$

where $\Gamma$ is the estimated Lorentzian linewidth.

\section{Results and discussion}

In Fig. 1(a) and (b) the evolution with temperature of the polarized and depolarized Raman spectra obtained from a Caf-loaded $\beta$-CDPMA14 hydrogel is shown in the wavenumber region between 1450 and $1850 \mathrm{~cm}^{-1}$.

As was previously reported ${ }^{14,29}$, the Raman spectra of NS hydrogels in this spectral region mainly display the vibrational signatures of the molecular moieties belonging to the PMA residues that compose the polymeric network of NS. ${ }^{14,38}$ A comparison between the Raman spectra of NS hydrogels with and without the addition of Caf (inset of Fig. 1(a)) suggests that the vibrational profiles of Caf-loaded NS in the spectral range of $1450-1850 \mathrm{~cm}^{-1}$ are mainly dominated by Raman signals arising from the polymer matrix.

The modes labelled in Fig. 1 (a) as $\nu(\mathrm{C}=\mathrm{C})_{1}, \nu(\mathrm{C}=\mathrm{C})_{2}$ and $\nu(\mathrm{C}=\mathrm{O})$ have been assigned to stretching motions involving the $\mathrm{C}=\mathrm{C}$ bonds of the aromatic rings of PMA units in the

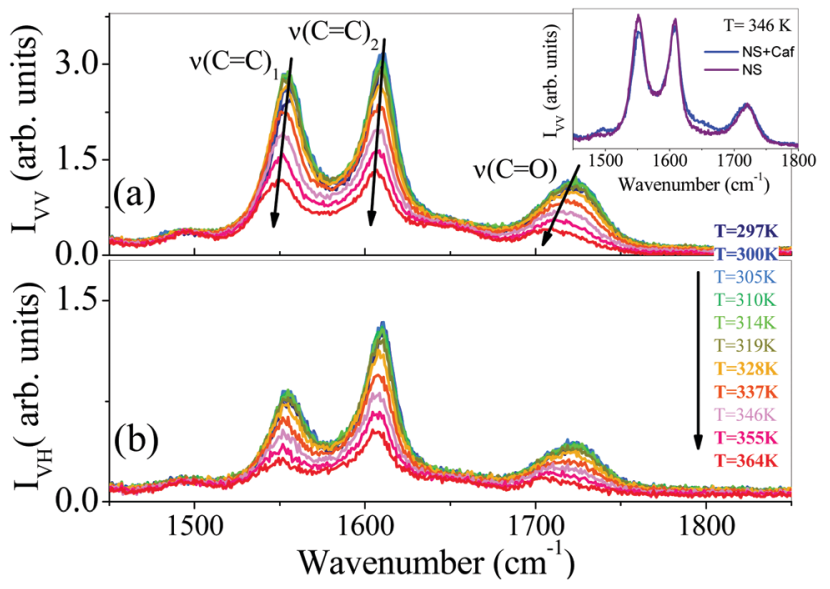

Fig. 1 Evolution with temperature of polarized (a) and depolarized (b) Raman spectra of $\beta$-CDPMA14 nanosponge loaded with Caf $\left(C_{\text {caf }}=80 \mathrm{mg} \mathrm{mL}^{-1}\right.$, $h=4)$. The arrows indicate the increase in temperature. Inset: Comparison between polarized Raman spectra of free and Caf-loaded NS hydrogels at $T=346 \mathrm{~K}$. 
polymeric network of $\mathrm{NS}\left(\nu(\mathrm{C}=\mathrm{C})_{1} \sim 1553 \mathrm{~cm}^{-1}\right.$ and $\nu(\mathrm{C}=\mathrm{C})_{2} \sim 1610 \mathrm{~cm}^{-1}$, respectively) and to stretching vibrations of carbonyl groups present in the polymer backbone $\left(\nu(\mathrm{C}=\mathrm{O}) \sim 1720 \mathrm{~cm}^{-1}\right) \cdot{ }^{13-15,29,38}$

The experimental spectra in Fig. 1 indicate a significant red shift in both polarized and depolarized Raman spectra in the Raman peaks $\nu(\mathrm{C}=\mathrm{C})_{1}, \nu(\mathrm{C}=\mathrm{C})_{2}$ and $\nu(\mathrm{C}=\mathrm{O})$ at high values of temperature $T$, together with a marked decrease in intensity. These spectral modifications suggest a change caused in the system by the increase in temperature, not only in terms of the rearrangement of molecular groups in the architecture of the polymer but also in terms of the breaking/reformation of intermolecular interactions.

The effect of temperature that was observed on the Raman spectra of NS hydrogels loaded with Caf was significantly more pronounced with respect to the temperature-induced modifications found for free NS hydrogels. ${ }^{29}$ In particular, the frequency and intensity of the Raman peak assigned to $\nu(\mathrm{C}=\mathrm{O})$ significantly changed with an increase in T in the spectra of NS with Caf. The opposite was found in the vibrational profiles of the same hydrogel without Caf, where this mode remained practically unchanged for all values of $T .^{29}$ In a similar way, the $\nu(\mathrm{C}=\mathrm{C})_{2}$ mode displays marked temperature-dependent modifications in Fig. 1, whereas only slight changes in the frequency of the same Raman peak were observed with an increase in $T$ in the case of the pristine hydrogel. ${ }^{29}$ This contrasting behaviour of the Raman modes associated with the NS matrix is consistent with the establishment of non-covalent interactions between Caf and molecular groups of the polymer backbone.

For a quantitative description of the response to $T$ described above, temperature-induced changes in the isotropic Raman spectra of NS loaded with Caf were studied, as shown in Fig. 2(a). Previous investigations of NS hydrogels ${ }^{14,29}$ have confirmed that the isotropic Raman profile is particularly informative on specific interactions established between the water solvent and the hydrophobic/hydrophilic groups of the NS polymer

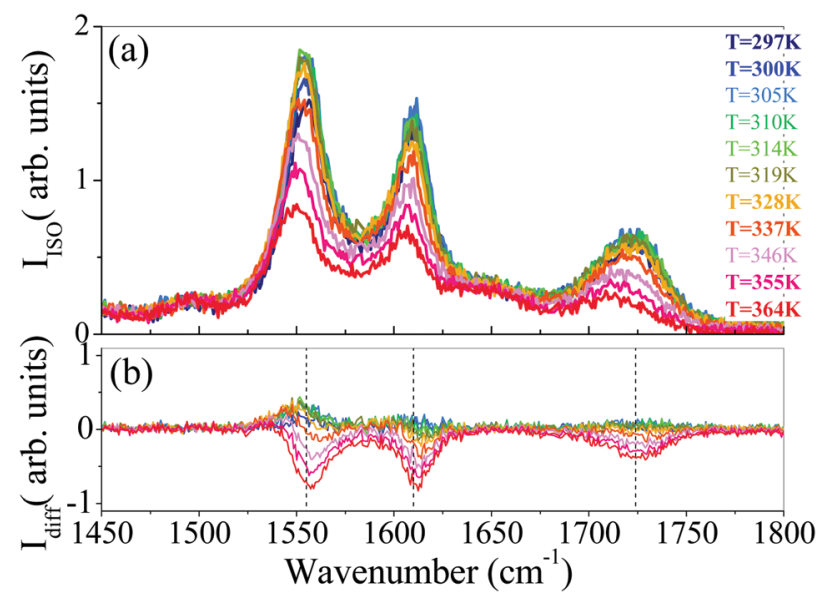

Fig. 2 (a) Evolution with temperature of isotropic Raman spectra of $\beta$-CDPMA14 nanosponge loaded with caffeine $\left(C_{\text {caf }}=83 \mathrm{mg} \mathrm{mL}^{-1}, h=4\right)$. (b) Temperature dependence of the difference spectral intensities $I_{\text {diff }}(T)$ obtained as reported in the text. via probing the vibrational relaxation processes occurring in the system. ${ }^{39,40}$

The effects of temperature on the spectral features of the isotropic Raman signals associated with the $\nu(\mathrm{C}=\mathrm{C})_{1}, \nu(\mathrm{C}=\mathrm{C})_{2}$ and $\nu(\mathrm{C}=\mathrm{O})$ modes can be clearly observed in Fig. 2(a), where the spectra obtained for the $\beta$-CDPMA14 hydrogel loaded with Caf are shown as a function of temperature, as an example. The spectral changes induced by an increase in temperature in the isotropic vibrational signals arising from the NS matrix are further visible by looking in Fig. 2(b) at the difference intensities $I_{\text {diff }}(T)$, which are estimated as:

$$
I_{\text {diff }}(T)=I_{\text {ISO }}(T)-I_{\text {ISO }} \quad(T=297 \mathrm{~K})
$$

where $I_{\mathrm{ISO}}(T)$ is the isotropic Raman spectrum acquired at temperature $T$ and $I_{\text {ISO }}(T=297 \mathrm{~K})$ is the experimental spectrum recorded at the lowest measured temperature of $T=297 \mathrm{~K}$.

As a general trend, the red shift observed in the modes assigned to the PMA residue suggests a strong modification of the local environment experienced by the probed oscillators upon a change in temperature in the hydrogel. In particular, the hydrophilic moieties, i.e., the $\mathrm{C}=\mathrm{O}$ groups of the polymeric network, seem to be less sensitive to intermolecular interactions with the environment at high $T$ values than the hydrophobic groups of the polymer. This is reflected by the following finding: the shift in frequency displayed by $\nu(\mathrm{C}=\mathrm{C})_{1}$ and $\nu(\mathrm{C}=\mathrm{C})_{2}(0.3 \%)$ was about one-half of the shift towards lower wavenumbers $(\sim 0.7 \%)$ observed for the $\nu(\mathrm{C}=\mathrm{O})$ mode. In the presence of Caf, the hydrophilic parts of the polymeric network displayed enhanced sensitivity to the breaking/formation of intermolecular interactions with the water solvent in comparison with the more hydrophobic moieties of NS (e.g., the aromatic rings of the crosslinker), which have been shown to be particularly responsive to changes in $\mathrm{pH}^{13,29}$ and hydration level. ${ }^{14}$

We have already reported ${ }^{29}$ how both hydrophobic and hydrophilic components play roles in determining the thermal activation of these hydrogels. The loading of Caf, which is a slightly hydrophilic molecule, would explain the enhanced sensitivity of the matrix towards water molecules.

The spectra shown in Fig. 2(b) indicate spectral modifications induced by $T$ in the peak shape for the $\nu(\mathrm{C}=\mathrm{C})_{1}$ mode. This change is expressed as a variation in the linewidth of the isotropic Raman peak associated with the $\nu(\mathrm{C}=\mathrm{C})_{1}$ vibration, which, at high temperatures, appears wider than at low $T$. In order to quantify the temperature-induced change in the peak shape for $\nu(\mathrm{C}=\mathrm{C})_{1}$, the same approach that was previously employed on samples of NS hydrogels without the presence of $\mathrm{Caf}^{14,29}$ was carried out.

Before fitting the isotropic Raman spectra (see Experimental section), a preliminary data treatment was needed in the case of the Caf-loaded hydrogels. This pretreatment was aimed at isolating the pure vibrational signal arising from the NS polymer component - namely, the Caf-free spectrum - from that derived from the contribution of Caf to the isotropic profile of the samples. In detail, the vibrational contribution originating from the guest molecule was removed from the total experimental intensity for the hydrogel loaded with Caf by subtracting the 


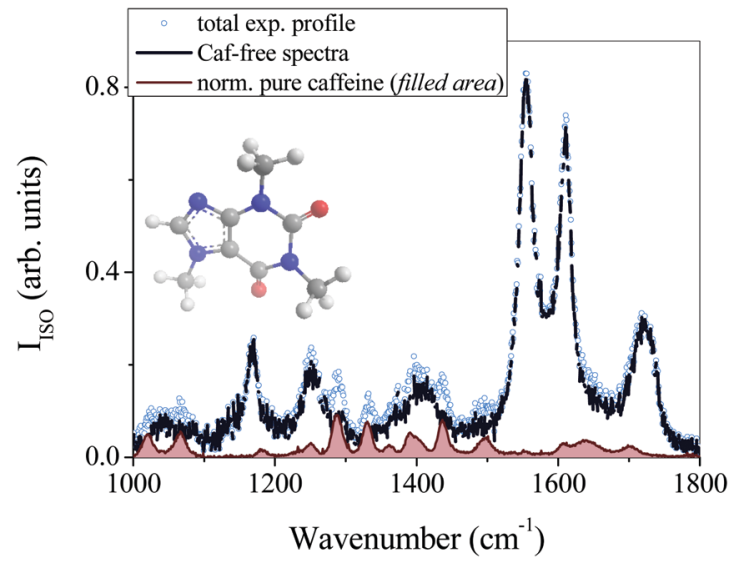

Fig. 3 Representative example of Caf-free isotropic profile obtained for $\beta$-CDPMA14 nanosponge loaded with Caf $\left(C_{\text {caf }}=83 \mathrm{mg} \mathrm{mL}^{-1}, h=4\right)$ : the total isotropic profile (empty symbols) is shown together with the normalized signal of pure caffeine (filled area) and the corresponding Caf-free spectrum that was obtained (continuous blue line). The sketch in the panel represents the chemical structure of Caf.

spectrum of pure Caf from the total after appropriate normalization of the spectrum on the intensity of the Raman signals found at 1019 and $1066 \mathrm{~cm}^{-1}$ and assigned to the vibrational modes of caffeine. The choice of the intensity of these two peaks as an internal standard for the normalization procedure was justified by the absence of Raman vibrational features associated with NS in the spectral range of $1000-1070 \mathrm{~cm}^{-1}$.

Fig. 3 shows an example of data treatment to obtain a Caffree isotropic profile after the subtraction procedure described above.

An inspection of Fig. 3 shows that the normalized vibrational contribution of Caf is almost negligible in the spectral range of $1500-1800 \mathrm{~cm}^{-1}$, where the peaks of NS under investigation are observed. This confirms that the described subtraction procedure did not significantly affect the final results.

Fig. 4 shows a typical example of best-fitted results obtained for the isotropic Raman Caf-free spectrum of the $\beta$-CDPMA14 hydrogel loaded with Caf, which was derived by using a combination of Kubo-Anderson functions, as reported in detail by Rossi et al. and D'Amico et al. ${ }^{14,29,39,40}$ As was expected, the Raman peaks assigned to the $\nu(\mathrm{C}=\mathrm{C})_{1}$ and $\nu(\mathrm{C}=\mathrm{C})_{2}$ modes were found to be satisfactorily well reproduced by a Lorentzian function. ${ }^{14,29}$ In this approximation, the corresponding dephasing time $\tau_{\text {deph }}$ can be computed from the estimated Lorentzian linewidth.

As already mentioned in previous works, ${ }^{39,40}$ the quantity $\left(1 / \tau_{\text {deph }}\right)$ can be interpreted, in hydrated systems, as a reasonable measurement of the rate of collision of solvent molecules with the vibrating chemical groups associated with the mode related to the dephasing time. In the specific case of NS hydrogels, ${ }^{14,29}$ the value of $\tau_{\text {deph }}$ associated with the $\nu(\mathrm{C}=\mathrm{C})_{1}$ mode was found to be a good indication of the dynamic restructuring of the interactions between water molecules trapped inside the hydrogel phase and hydrophobic molecular groups present in the polymeric network of NS.

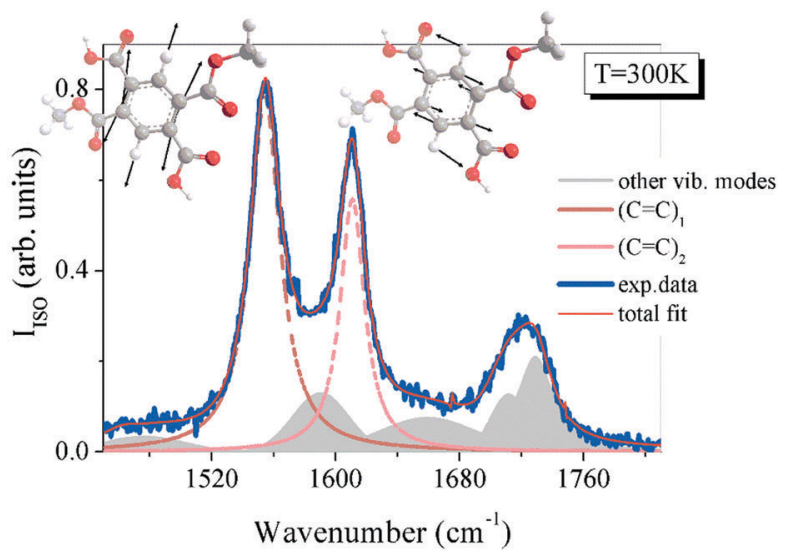

Fig. 4 Typical example of best-fitted results for isotropic Raman Caf-free spectrum of $\beta$-CDPMA14 nanosponge loaded with caffeine $\left(C_{\text {caf }}=83 \mathrm{mg} \mathrm{mL}^{-1}\right.$, $h=4$ ). The total fit curve (orange line) is shown together with the experimental spectrum (blue line) and the single spectral components, as indicated in the panel. The sketches in the graph illustrate the vibrational modes associated with the Raman signals for $\nu(\mathrm{C}=\mathrm{C})_{1}$ and $\nu(\mathrm{C}=\mathrm{C})_{2}$.

Fig. 5 shows the temperature dependence of the dephasing time $\tau_{\text {deph }}$ associated with the $\nu(\mathrm{C}=\mathrm{C})_{1}$ mode of the PMA residues of the polymeric network of NS, which was estimated for NS hydrogels that were both pure and loaded with Caf at different final concentrations.

The data displayed in Fig. 5 show the trend that was already observed in the dephasing time associated with the $\nu(\mathrm{C}=\mathrm{C})_{1}$ mode, and the value of $\tau_{\text {deph }}$ remains substantially temperatureindependent up to an activation temperature, which is indicated as $T^{*}$. For $T>T^{*}$, the dephasing time tends to decrease linearly. In the panel of Fig. 5 the point corresponding to the activation temperature $T_{\mathrm{Ns}}{ }^{*}$ that was estimated for the pure $\beta$-CDPMA14 hydrogel ${ }^{29}$ is highlighted by an arrow, as an example. Similar behaviour with temperature of the dephasing time was found both for the pristine hydrogel and for all the samples loaded with Caf, as shown in Fig. 5. This can be interpreted,

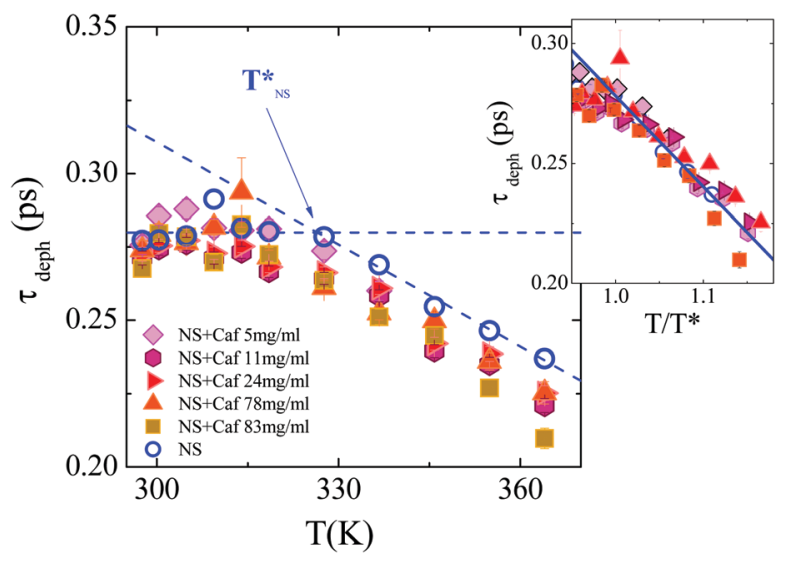

Fig. 5 Evolution with temperature of the dephasing time $\tau_{\text {deph }}$ associated with the $\nu(C=C)_{1}$ Raman mode for the $\beta$-CDPMA14 hydrogel (data from Rossi et al. ${ }^{29}$ ) and $\beta$-CDPMA14 loaded with caffeine at different final concentrations $(h=4)$. Inset: Plot of the dephasing times scaled by the estimated values of $T^{\star}$ for each hydrogel sample (see text for details). 
Table 1 Values of the activation temperature $\left(T^{\star}\right)$ estimated for $\beta$-CDPMA14 loaded with Caf at different concentrations $(h=4)$

\begin{tabular}{lll}
\hline $\begin{array}{l}\text { Concentration of } \\
\text { caffeine } C_{\text {caf }}(\mathrm{mg} \mathrm{mL}\end{array}$ & & \\
\hline 0 & Caf/NS $(\mathrm{w} / \mathrm{w})$ & Estimated $T^{*}(\mathrm{~K})$ \\
5 & 0 & $328.0 \pm 1$ \\
11 & 0.02 & $317.9 \pm 1$ \\
24 & 0.044 & $316.3 \pm 1$ \\
78 & 0.096 & $315.8 \pm 1$ \\
83 & 0.312 & $312.3 \pm 1$ \\
& 0.332 & $313.0 \pm 1$ \\
\hline
\end{tabular}

from a molecular point of view, in terms of an increase in the rate of collision between water molecules and hydrophobic $\mathrm{CH}$ groups on PMA moieties in the polymeric network of NS, which occurs at temperatures greater than the triggering temperature $T^{*}$. This finding has been in turn connected to stronger solvation of the more hydrophobic moieties of the polymer backbone ${ }^{29}$ at $T>T^{*}$. Therefore, the activation temperature $T^{*}$ can be thought of as a physical indicator of a change in the hydrophobic/hydrophilic character of the chemical moieties on PMA residues in the polymeric network of NS, which is dependent on variations in conditions of the system such as the $\mathrm{pH}^{29}$

Interestingly, the data in Fig. 5 indicate that the values of the activation temperature $T^{*}$ obtained for NS hydrogels with and without Caf are rather different, as shown by the values of $T^{*}$ reported in Table 1.

As a general trend, a significant reduction $(\sim 10 \mathrm{~K})$ in the activation temperature $T^{*}$ was observed as a consequence of the addition of Caf to the polymeric gel. This finding confirms the hypothesis proposed above that the host-guest interactions established between Caf and the polymeric network of NS strongly affect the thermoresponsive behaviour exhibited by nanosponge hydrogels. In particular, the decrease in $T^{*}$ can be explained by proposing a model in which the loading of Caf into NS hydrogels tends to favour access of the water solvent to the more hydrophobic $\mathrm{CH}$ groups of the NS matrix, which in turn results in more marked solvation of the whole system.

In order to better account for the linear decrease in $\tau_{\mathrm{deph}}$ with $T$ for $T>T^{*}$, the plots in Fig. 5 were rescaled in terms of $T / T^{*}$, as shown in the inset of Fig. 5. Following this procedure, the data for $\tau_{\mathrm{deph}}\left(\frac{T}{T^{*}}\right)$ that correspond to the different samples of NS hydrogels merged into a single master curve (see the inset of Fig. 5). The existence of this master curve highlights that the rate of decrease observed in $\tau_{\mathrm{deph}}$ at $T>T^{*}$ is essentially the same for the pure NS gel and NS loaded with Caf. Thus, NS hydrogels experienced the same thermoresponsive mechanism in the native and Caf-loaded phases, but triggered at different activation temperatures $T^{*}$ by the addition of the guest molecule to the hydrogel.

Fig. 6 shows the dependence of the activation temperature $T^{*}$ on the guest concentration and $\mathrm{pH}$ measured for the two different types of NS hydrogels loaded with Caf.

The data shown in panel (a) of Fig. 6 confirm better the decrease in $T^{*}$ triggered by the loading of the guest molecule into the NS gel. Once again, we could ascribe this behaviour to an increase in the hydrophilic component of the matrix owing

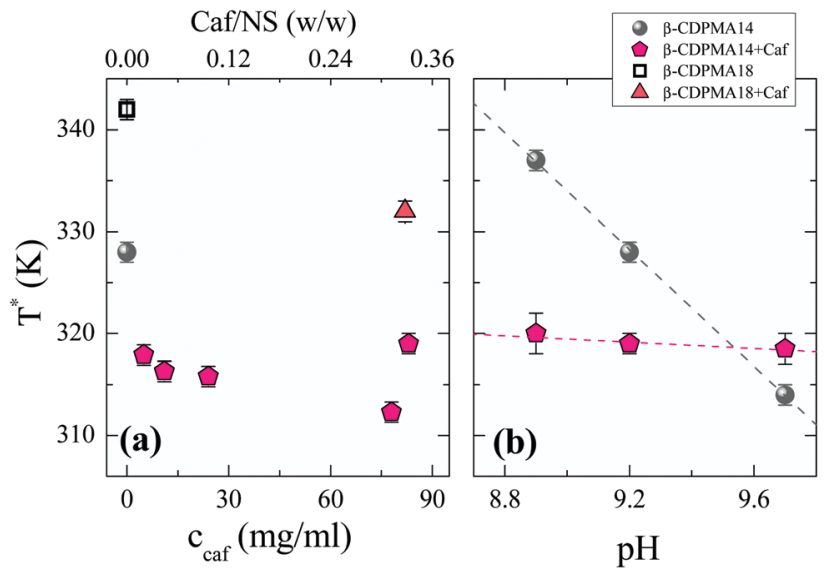

Fig. 6 (a) Dependence on caffeine concentration of estimated value of $T^{\star}$ for $\beta$-CDPMA14 and $\beta$-CDPMA18 nanosponge hydrogels loaded with Caf and hydrated in the same conditions $(h=4)$. The concentration of Caf is reported both with respect to the hydrating solution $\left(C_{\text {caf }}\right)$ and to the amount of NS (Caf/NS w/w). The full circles and open squares represent the activation temperature $T^{*}$ measured for the pure $\beta$-CDPMA14 and $\beta$-CDPMA18 hydrogels, respectively. (b) Evolution with $\mathrm{pH}$ of $T^{\star}$ for $\beta$-CDPMA14 nanosponge hydrogels in the pure state (full circles) and loaded with Caf at $C_{\text {caf }}=80 \mathrm{mg} \mathrm{mL}^{-1}$ (pink diamonds). The dotted lines are linear fits of the experimental data.

to the slightly polar character of Caf. This behaviour is also found when the molecular architecture of NS is changed, as in the case of the $\beta$-CDPMA18 formulation. The lesser effect of the addition of Caf on the decrease in $T^{*}$ for the latter NS gel seems to support our hypothesis. In $\beta$-CDPMA18 polymers, the hydrophilic component is more significant than in $\beta$-CDPMA14 owing to the presence of a larger amount of free carboxylic moieties. As a consequence, the addition of Caf is not expected to affect significantly the hydrophilic character of the hydrogel.

The data for $T^{*}$ shown in Fig. 6(a) also confirm the slight dependence on the concentration of Caf of the activation temperature measured for the gel, which changed from about 317 to $313 \mathrm{~K}$ when $C_{\text {caf }}$ increased from 5 to $83 \mathrm{mg} \mathrm{mL}^{-1}$.

Finally, the plots in Fig. 6(b) show a comparison between the dependence on $\mathrm{pH}$ of the activation temperature $T^{*}$ estimated for $\beta$-CDPMA14 hydrogel in the pure state and loaded with fixed concentrations of Caf. Previous results confirmed a marked dependence on $\mathrm{pH}$ of $T^{*}$ for NS hydrogels ${ }^{29}$ (see full circles shown in Fig. 6(b)). This has been explained by an enhancement in the slightly acidic behaviour of the $\mathrm{CH}$ groups on the aromatic rings of PMA owing to the $\mathrm{pH}$ of the surrounding aqueous medium, which was in turn reflected in a significant exposure of these hydrophobic sites to collision with water molecules. Interestingly, the data for $T^{*}$ obtained at different $\mathrm{pH}$ values for NS loaded with Caf provide evidence that the addition of this guest molecule seems to overcome the effects of $\mathrm{pH}$ on the hydrophobic character of the $\mathrm{CH}$ groups of PMA. In other words, the predominant hydrophilic component of Caf-loaded NS gel is already evident at a lower $\mathrm{pH}$. In fact, the loaded matrix at $\mathrm{pH}=8.9$ exhibits a $T^{*}$ value analogous to that measured at $\mathrm{pH}=9.7$ for both loaded (pink diamonds) and Caf-free NS gels (full circles in Fig. 6(b)). This is reflected in the practically negligible dependence on $\mathrm{pH}$ observed for $T^{*}$. 
Recent studies performed on $\beta$-CDPMA14 gel ${ }^{13}$ confirmed that the increase in the swelling capability of NS that was experimentally observed with an increase in the $\mathrm{pH}$ of the gel phase $\mathrm{e}^{29}$ could be consistent with a possible increase in the mesh size of the hydrogel taking place at high $\mathrm{pH}$ values in NS hydrogels. However, this structural modification of the polymeric network of NS does not seem to significantly affect the value of $T^{*}$ in the case of Caf-loaded gels, in contrast to pure NS. This surprising result confirms the predominant role played by guest-host interactions with respect to more definite structural effects in governing the loading and diffusion of Caf inside NS gels.

\section{Conclusions}

The UV Raman spectra of hydrogels were analysed in depth as a function of temperature, concentration of the guest molecule loaded in the gel phase, and $\mathrm{pH}$, because it has been demonstrated that they are particularly informative, both on the structural rearrangements of the hydrophobic/hydrophilic groups of the polymeric network and on the breaking/formation of specific non-covalent interactions in the system.

As the main result, the addition of Caf as a guest component inside the polymeric gel of NS was found to cause a significant decrease in the value of the activation temperature $T^{*}$ measured for the hydrogel. This descriptor quantitatively accounts for the change in the hydrophobic/hydrophilic character of the chemical moieties on PMA residues in the polymeric network of NS, which is in turn connected with the solvation behaviour of the whole system. As a possible rationale for these findings, we propose a molecular model in which the loading of Caf in NS hydrogels tends to favour access of the water solvent to the more hydrophobic $\mathrm{CH}$ groups of the NS matrix as a direct consequence of the host-guest interactions established in the system. All these results provide significant new insights into our knowledge of NS hydrogels, which, until now, was limited to the investigation of the water-polymer interactions in the gel phase. This appears, in turn, to be of interest with respect to the design of possible strategies for controlling the diffusion and release rate of bioactive molecules inside hydrogel networks. In addition, the achievements of this work corroborate the potential of UV Raman scattering experiments to give new molecular insights into complex phenomena affecting hydrogel phases.

\section{Acknowledgements}

The authors acknowledge the CERIC-ERIC Consortium for the access to experimental facilities and financial support. Regione Toscana (POR FESR 2014-2020, Call RSI 2014, Project NanoBonD) is also acknowledged for financial support.

\section{Notes and references}

1 O. Wichterle and D. Lim, Nature, 1960, 185, 117.

2 N. A. Peppas, B. V. Slaughter, M. A. Kanzelberger, in Hydrogels, in Comprehensive Polymer Science, ed. R. Langer and D. Tirrell, Elsevier, 2011, vol. 9.
3 E. H. Schacht, J. Phys.: Conf. Ser., 2004, 3, 22.

4 W. E. Hennink and C. F. van Nostrum, Adv. Drug Delivery Rev., 2002, 54, 13.

5 T. T. Lau and D. A. Wang, Nanomedicine, 2013, 8(4), 655.

6 M. W. Tibbitt and K. S. Anseth, Biotechnol. Bioeng., 2009, 103(4), 655.

7 A. M. Greiner, M. Jäckel, A. C. Scheiwe, D. R. Stamowd, T. J. Autenrieth, J. Lahann, C. M. Franz and M. Bastmeyer, Biomaterials, 2014, 35, 611.

8 H. J. Yoo and H. D. Kim, J. Biomed. Mater. Res., Part B, 2008, 85, 326.

9 J. Chen, H. Park and K. Park, J. Biomed. Mater. Res., 1999, 44, 53.

10 A. Matsumoto, T. Ishii, J. Nishida, H. Matsumoto, K. Kataoka and Y. Miyahara, Angew. Chem., Int. Ed., 2012, 51, 2124.

11 A. K. A. Silva, C. Richard, M. Bessodes, D. Scherman and O. W. Merten, Biomacromolecules, 2008, 10, 9.

12 T. Tanigo, R. Takaoka and Y. Tabata, J. Controlled Release, 2010, 143, 201.

13 B. Rossi, V. Venuti, A. Mele, C. Punta, L. Melone, F. D’Amico, A. Gessini, V. Crupi, D. Majolino, F. Trotta and C. Masciovecchio, Phys. Chem. Chem. Phys., 2016, 18, 12252.

14 B. Rossi, V. Venuti, F. D’Amico, A. Gessini, F. Castiglione, A. Mele, C. Punta, L. Melone, V. Crupi, D. Majolino, F. Trotta and C. Masciovecchio, Phys. Chem. Chem. Phys., 2015, 17, 963.

15 B. Rossi, V. Venuti, A. Mele, C. Punta, L. Melone, V. Crupi, D. Majolino, F. Trotta, F. D'Amico, A. Gessini and C. Masciovecchio, J. Chem. Phys., 2015, 142(1), 014901.

16 N. A. Peppas, P. Bures, W. Leobandung and H. Ichikawa, Eur. J. Pharm. Biopharm., 2000, 50, 27.

17 B. Jeong, S. Kim and Y. Bae, Adv. Drug Delivery Rev., 2002, $\mathbf{5 4}, 37$.

18 T. Miyata, T. Uragami and K. Nakamae, Adv. Drug Delivery Rev., 2002, 54, 79.

19 N. A. Peppas and A. R. Khare, Adv. Drug Delivery Rev., 1993, $11,1$.

20 T. R. Hoare and D. S. Kohane, Polymer, 2008, 49, 1993.

21 J. L. Peng Soh, C. V. Liew and P. W. Sia Heng, Curr. Pharm. Des., 2015, 21(40), 5890.

22 F. Trotta, M. Zanetti and R. Cavalli, Beilstein J. Org. Chem., 2012, 8, 2091.

23 S. V. Chilajwar, P. P. Pednekar, K. R. Jadhav, G. J. C. Gupta and V. J. Kadam, Expert Opin. Drug Delivery, 2014, 11(1), 111.

24 R. Z. Ahmed, G. Patil and Z. Zaheer, Drug Dev. Ind. Pharm., 2013, 39(9), 1263.

25 G. Tejashiri, B. Amrita and J. Darshana, Acta Pharm., 2013, 63, 335.

26 (a) F. Trotta, C. Dianzani, F. Caldera, B. Mognetti and R. Cavalli, Expert Opin. Drug Delivery, 2014, 11(6), 931; (b) V. Venuti, B. Rossi, A. Mele, L. Melone, C. Punta, D. Majolino, C. Masciovecchio and F. Caldera, Expert Opin. Drug Delivery, 2016, DOI: 10.1080/17425247.2016.1215301.

27 L. Seglie, K. Martina, M. Devacchi, C. Roggero, F. Trotta and V. Scariot, Postharvest Biol. Technol., 2011, 59, 200. 
28 D. Li and M. Ma, Clean Products and Processes, 2000, vol. 2, p. 112.

29 B. Rossi, V. Venuti, F. D’Amico, A. Gessini, A. Mele, C. Punta, L. Melone, V. Crupi, D. Majolino, F. Trotta and C. Masciovecchio, Soft Matter, 2015, 11, 5862.

30 V. Crupi, D. Majolino, A. Mele, L. Melone, C. Punta, B. Rossi, F. Toraldo, F. Trotta and V. Venuti, Soft Matter, 2014, 10, 2320.

31 M. Ferro, F. Castiglione, C. Punta, L. Melone, W. Panzeri, B. Rossi, F. Trotta and A. Mele, Beilstein J. Org. Chem., 2014, 10, 2715.

32 T. Ranheim and B. Halvorsen, Mol. Nutr. Food Res., 2005, 49, 274.

33 R. M. van Dam, Appl. Physiol., Nutr., Metab., 2008, 33, 1269.

34 D. Hexsel, C. Orlandi and D. Zechmeister do Prado, Dermatol. Surg., 2005, 31, 866.
35 M. A. Bolzinger, S. Briançon, J. Pelletier, H. Fessi and Y. Chevalier, Eur. J. Pharm. Biopharm., 2008, 68, 446.

36 D. Cunha, M. B. Yahia, S. Hall, S. R. Miller, H. Chevreau, E. Elkaïm, G. Maurin, P. Horcajada and C. Serre, Chem. Mater., 2013, 25, 2767.

37 F. D’Amico, M. Saito, F. Bencivenga, M. Marsi, A. Gessini, G. Camisasca, E. Principi, R. Cucini, S. DiFonzo and A. Battistoni, Nucl. Instrum. Methods Phys. Res., Sect. A, 2013, 703, 33.

38 F. Castiglione, V. Crupi, D. Majolino, A. Mele, B. Rossi, F. Trotta and V. Venuti, J. Phys. Chem. B, 2012, 116(27), 7952.

39 F. D’Amico, F. Bencivenga, G. Gessini, E. Principi, R. Cucini and C. Masciovecchio, J. Phys. Chem. B, 2012, 116, 13219.

40 F. D’Amico, F. Bencivenga, G. Camisasca, A. Gessini, E. Principi, R. Cucini and C. Masciovecchio, J. Chem. Phys., 2013, 139, 015101. 